\title{
physica status solidi
}

年 


\section{Classification Scheme}

1. Structure of Solids

1.1 Alloys. Metallurgy

1.2 Solid-State Phase Transformations

1.3 Surfaces

1.4 Films

2. Non-Crystalline State

3. Crystallography

3.1 Crystal Growth

3.2 Interatomic Forces

4. Microstructure of Solids

5. Perfectly Periodic Structures

6. Lattice Mechanics. Phonons

6.1 Mössbauer Investigations

7. Acoustic Properties of Solids

8. Thermal Properties of Solids

9. Diffusion in Solids

10. Defect Properties of Solids (Irradiation Defects see 11)

10.1 Defect Properties of Metals

10.2 Photochemical Reactions. Colour Centres

11. Irradiation Effects in Solids

12. Mechanical Properties of Solids (Plastic Deformations see 10)

12.1 Mechanical Properties of Metals (Plastic Deformations see 10.1)

13. Electron States in Solids

13.1 Band Structure. Fermi Surfaces

13.2 Excitons

13.3 Surface States

13.4 Impurity and Defect States

14. Electrical Properties of Solids. Transport Phenomena

14.I Metals. Conductors

14.2 Superconductivity. Superconducting Materials and Devices

14.3 Semiconductors

14.3.1 Semiconducting Films

14.3.2 Semiconducting Devices. Junctions (Contact Problems see 14.4.1)

14.4 Dielectrics

14.4.1 High Field Phenomena, Space Charge Effects, Inhomogeneities, Injected

Carriers (Electroluminescence see 20.3; Junctions see 14.3.2)

14.4.2 Ferroelectric Materials and Phenomena

15. Thermoelectric and Thermomagnetic Properties of Solids

16. Photoconductivity. Photovoltaic Effects

17. Emission of Electrons and Ions from Solids

18. Magnetic Properties of Solids

18.1 Paramagnetic Properties

18.2 Ferromagnetic Properties

18.3 Ferrimagnetic Properties. Ferrites

18.4 Antiferromagnetic Properties 


\title{
physica status solidi
}

\author{
Board of Editors
}

P. AIGRAIN, Paris, S. AMELINCKX, Mol-Donk,

V. L. BONCH-BRUEVICH, Moskva, W. DEKEYSER, Gent,

W. FRANZ, Münster, P. GÖRLICH, Jena, E. GRILLOT, Paris, R. KAISCHEW, Sofia, P. T. LANDSBERG, Cardiff, L. NEEL, Grenoble,

A. PIEKARA, Warszawa, A. SE EGER, Stuttgart, F. SEITZ, Urbana, O.STASIW, Berlin, M. STEENBECK, Jena,

F. STÖCKM ANN, Karlsruhe, G. SZIGETI, Budapest, J. TAUC, Praha

\section{Editor-in-Chief \\ P. GÖRLICH \\ Advisory Board}

M. BALKANSKI, Paris, P. C. BANBURY, Reading, M. BERNARD, Paris, W. BRAUER, Berlin, W. COCHRAN, Edinburgh, R. COELHO, Fontenay-aux-Roses, H.-D. D I T T ZE, Saarbrücken, J.D. ESHEL BY, Cambridge, P. P. F E O F I L O V, Leningrad, J. HOPFIELD, Princeton, G. J A COBS, Gent, J. JA UMANN, Köln, E. KLIER, Praha,

E. KROENER, Clausthal-Zellerfeld, R. KUBO, Tokyo, M. MATYÁŠ, Praha,

H. D. MEGAW, Cambridge, T. S. MOSS, Camberley, E. NAGY, Budapest,

E. A. NIEKISCH, Jülich, L. PAL, Budapest, M. RODOT, Bellevue/Seine,

B. V. ROLLIN, Oxford, H. M. ROSENBERG, Oxford, R. VAUTIER, Bellevue/Seine

Volume $28 \cdot$ Number $2 \cdot$ Pages 429 to $850, \mathrm{~K} 97$ to K182, and A5 to A10

August 1, 1968

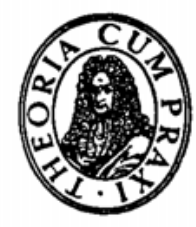

A K A D E M I E - V E R LA G - B E R L I N 
Subscriptions and orders for single copies should be addressed to

AKADEMIE.VERLAG GmbH, 108 Berlin, Leipziger Straße 3-4 or to Buchhandlung KUNST UND WISSEN, Erich Bieber, 7 Stuttgart 1, Wilhelmstr. 4 - 6 or to Deutsche Buch-Export und-Import GmbH, 701 Leipzig, Postschließfach 160

\section{Editorial Note:}

"physica status solidi" undertakes that an original paper accepted for publication before the $8^{\text {th }}$ of any month will be published within 50 days of this date unless the author requests a postponement. In special cases there may be some delay between receipt and acceptance of a paper due to the review and, if necessary, revision of the paper.

Schriftleiter und verantwortlich für den Inhalt: Professor Dr. Dr. h. c. P. G örli ch, 102 Berlin, Neue Schönhauser Str. 20 bzw. 69 Jena, Humboldtstr. 26. Redaktionskollegium: Dr. S. Oberländer, Dr. E. Gutsche, Dr. W. Borchardt. Anschrift der Schriftleitung: 102 Berlin, Neue Schōnhauser Str. 20, Fernruf: 426788 . Verlag: Akademie-Verlag GmbH 108 Berlin, Leipziger Str. 3-4. Fernruf: 220441, Telex-Nr. 112 020. Postscheckkonto: Berlin $35021 .-$ Die Zeitschrift "physica status solidi"* erscheint jeweils am 1. des Monats. Bezugspreis eines Bandes M 90, (Sonderpreis für die DDR M 60,-). Bestellnummer dieses Bandes 1068/28. Jeder Band enthält zwei Hefte. Gesamtherstellung: VEB Druckerei „Thomas Müntzer" Bad Langensalza.- Veröffentlicht unter der Lizenznummer 1310 des Presseamtes beim Vorsitzenden des Ministerrates der Deutschen Demokratischen Republik. 